\title{
Palmaris Longus Tendon Graft as Docking For Restablisation of Lateral Collateral Elbow Injury
}

\author{
${ }^{1}$ Dr Birendra Kumar, ${ }^{2}$ Dr N.D.Kachhap, ${ }^{3}$ Dr Devendra kumar, \\ ${ }^{4}$ Dr Sundeep Kumar Sunkesula, (DNB), ${ }^{5}$ Dr Abhishek Parashar (DNB) \\ ${ }^{I}$ Specialist, Dept of Ortho.(main author)BGH Bokaro \\ ${ }^{2}$ HOD .Dept of Ortho, BGH Bokaro \\ ${ }^{3} \mathrm{Sr}$ consultant, dept of Ortho, BGH Bokaro \\ ${ }^{4,5}$ Dept of Ortho, BGH Bokaro
}

\section{Introduction}

- Post. dislocation injuries are common causes of lateral collateral ligament injury due to high energy trauma.

- Elbow collateral ligament insufficiency is commonly seen in sports participants involved in over-arm throwing sports.

- The lateral ligament prevents rotational instability between the distal humerus and the proximal radius and ulna.

- LCL injury can result in elbow varus instability, supination and flexion affected.

- Jobe et al first described double-strand reconstruction of the lateral collateral ligament (LCL) with use of a free tendon graft that was secured to the lateral epicondyle and the proximal aspect of the radius in a figureeight fashion.

\section{Anatomy}

- lateral collateral ligament complex capsuloligamentous structure, consisting of four ligaments:

1.Radial collateral ligament (RCL)

2. Lateral ulnar collateral ligament (LUCL)

3. Annular ligament (AL)
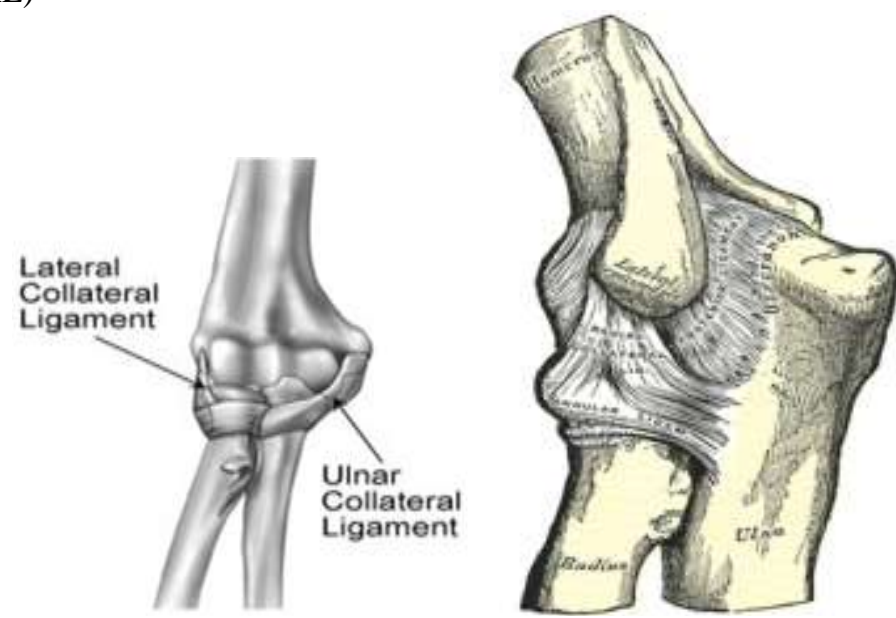

- Injury to the lateral collateral ligament (LCL) is a consistent feature of traumatic elbow instability and inadequate treatment of the LCL is the source of a large proportion of the cases of posttraumatic elbow instability.

- The LCL is far more important than the MCL in the setting of most cases of traumatic elbow instability. The trochlear notch (coronoid and olecranon), radial head and LCL are repaired or reconstructed but the MCL rarely needs to be repairedity.(Rockwood Green)

\section{Classification}

- O'Driscoll has described three stages of posterolateral rotatory elbow instability .

- Stage 1-Involves partial or complete disruption of the LCL.

- Stage 2- Involves an incomplete posterior dislocation with disruption of the lateral ligamentous complex and further injury to the osseous or ligamentous structure.

- $\quad$ Stage 3 - This includes LCL \& MCL with complete posterior dislocation. 


\section{Normal MRI picture}
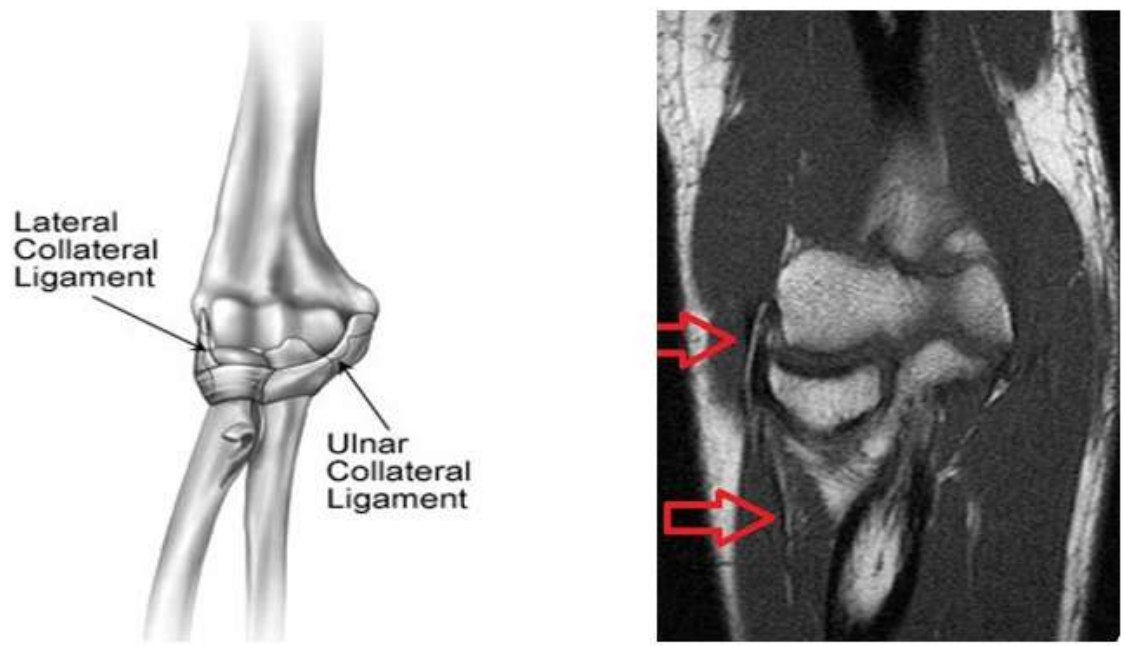

\section{Diagnosis}

- History and high index suspicion

- Clinical examination - stress test

- Radiology - X-ray may suggestive of capsuloligamentous -osseous involvment

- $\quad$ MRI

- Avulsion of lat. Coll. Lig.

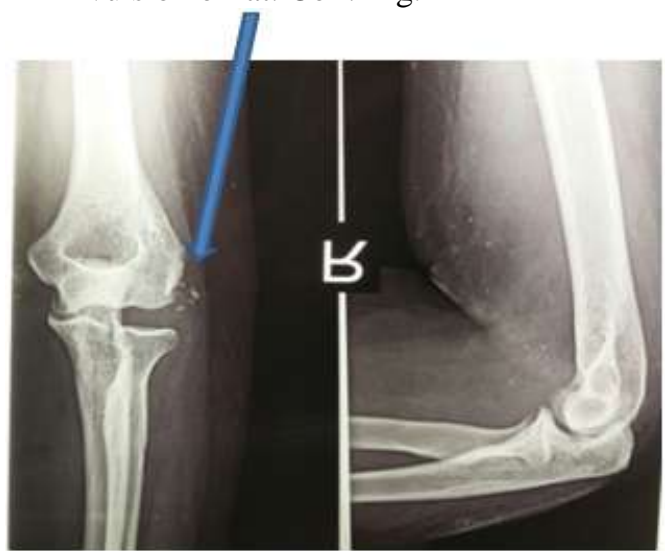

MRI - Axial and coronal fat sat images showing marrow contusion in lateral condyle humerus and lat. Coll. ligament as focal increased signals

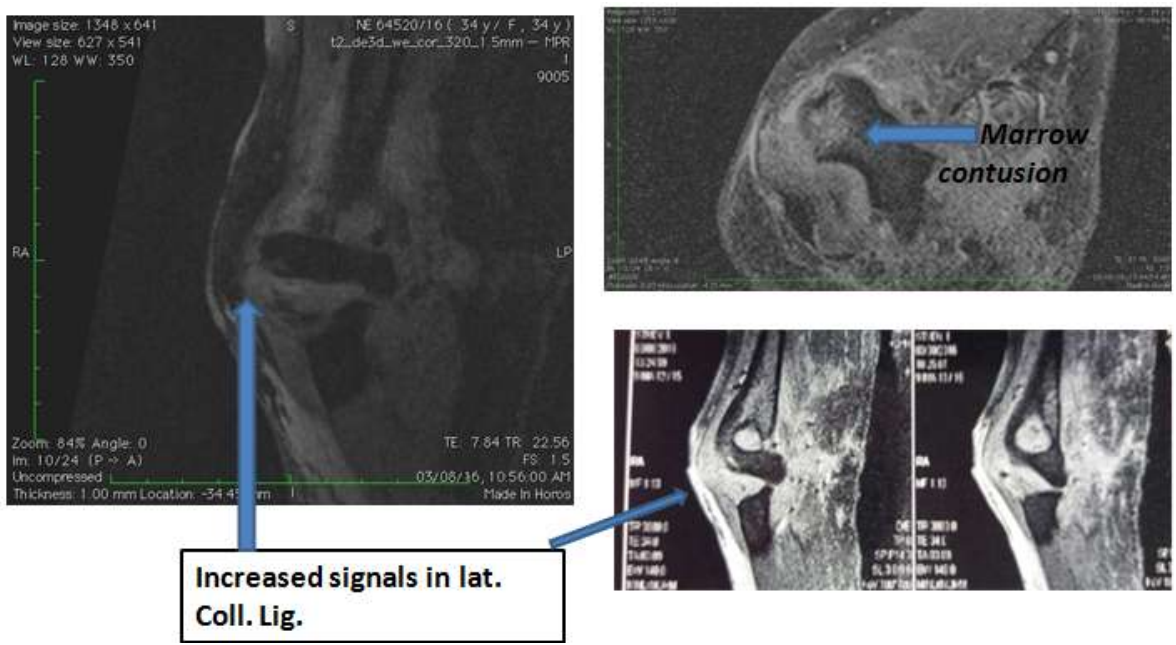


Clinical diagnosis

Positive varus stress test suggestive of lat. coll. disruption

Negative valgus stress test suggestive of intact med. Coll.

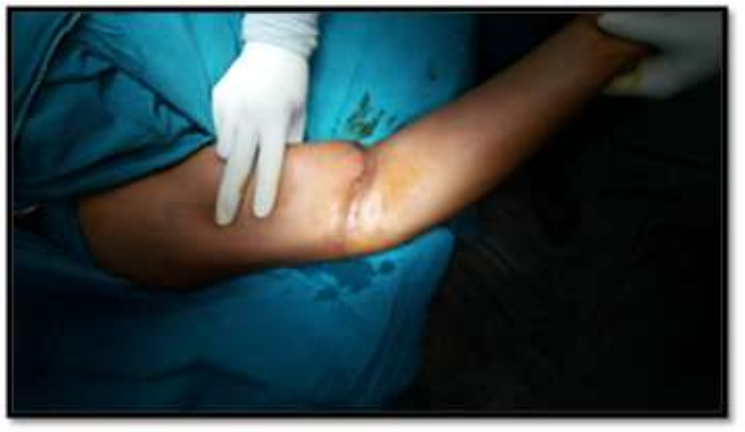

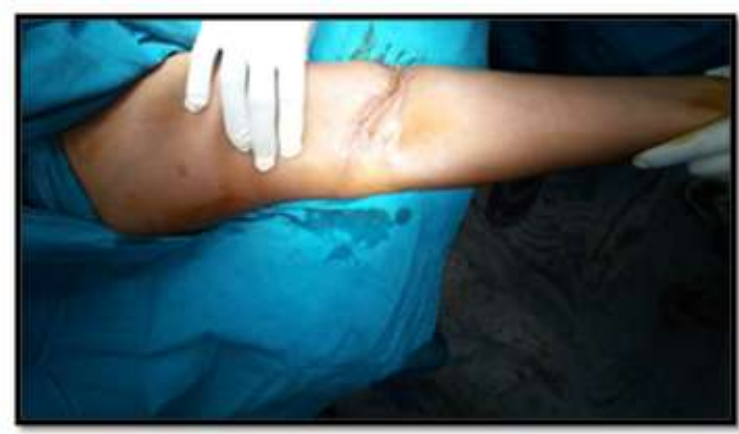

Healed scar over anterior aspect of elbow

Intraoperative findings

- Lacerated lateral ligament support with clinical radial head subluxation

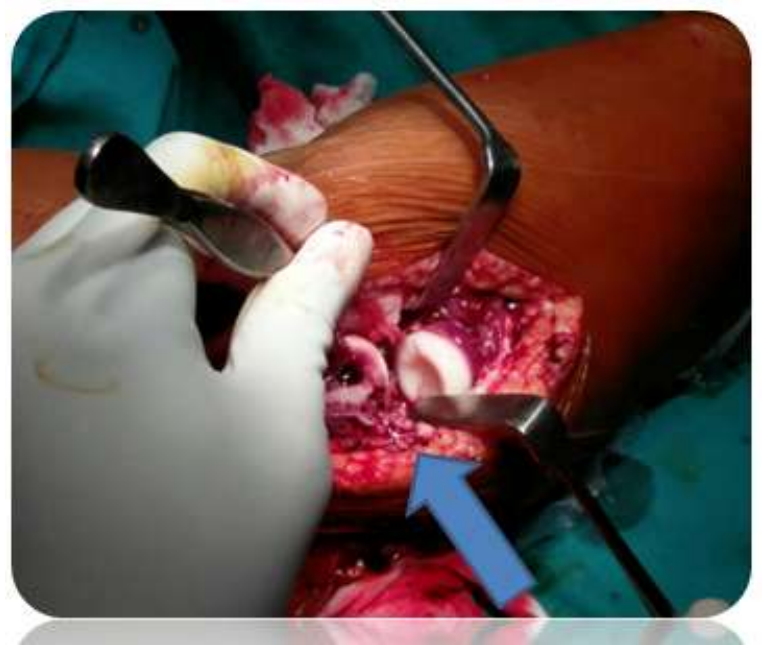

Harvesting palmaris longus graft
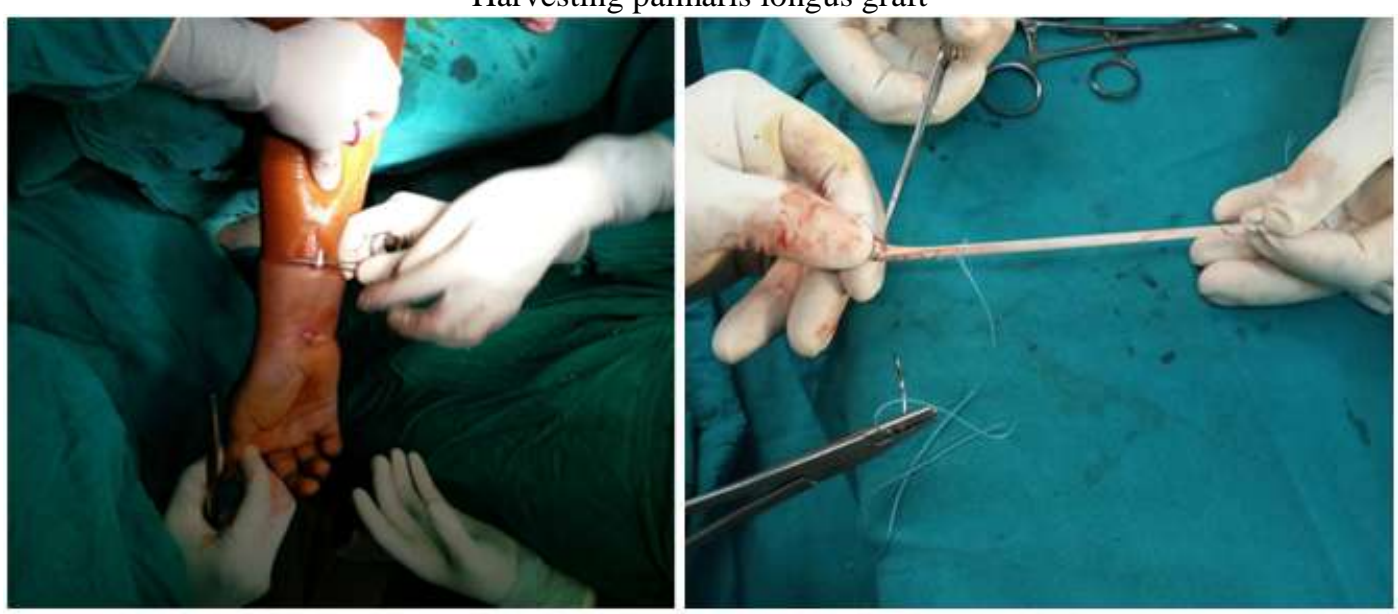

Intra-operative procedure

- Palmaris graft application from radial neck to lateral condyle 


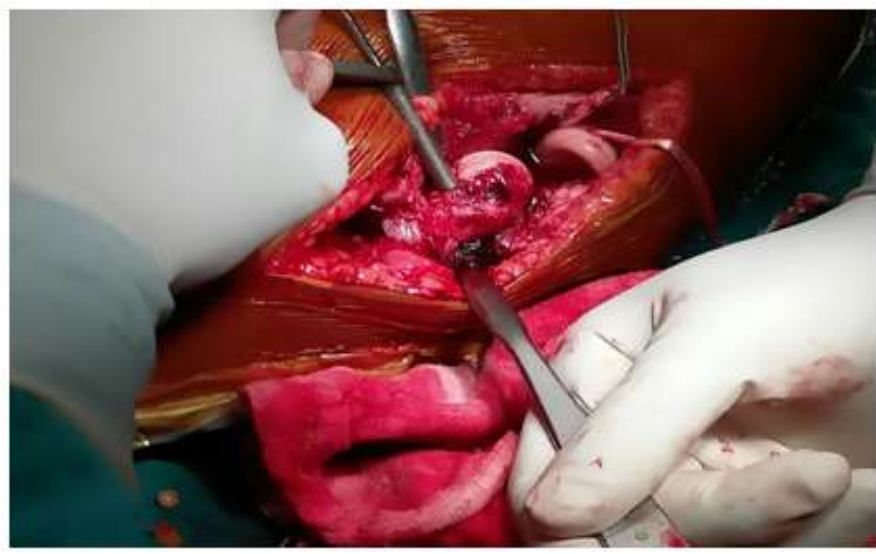

Intra-operative final tie-up in figure 8 fashion
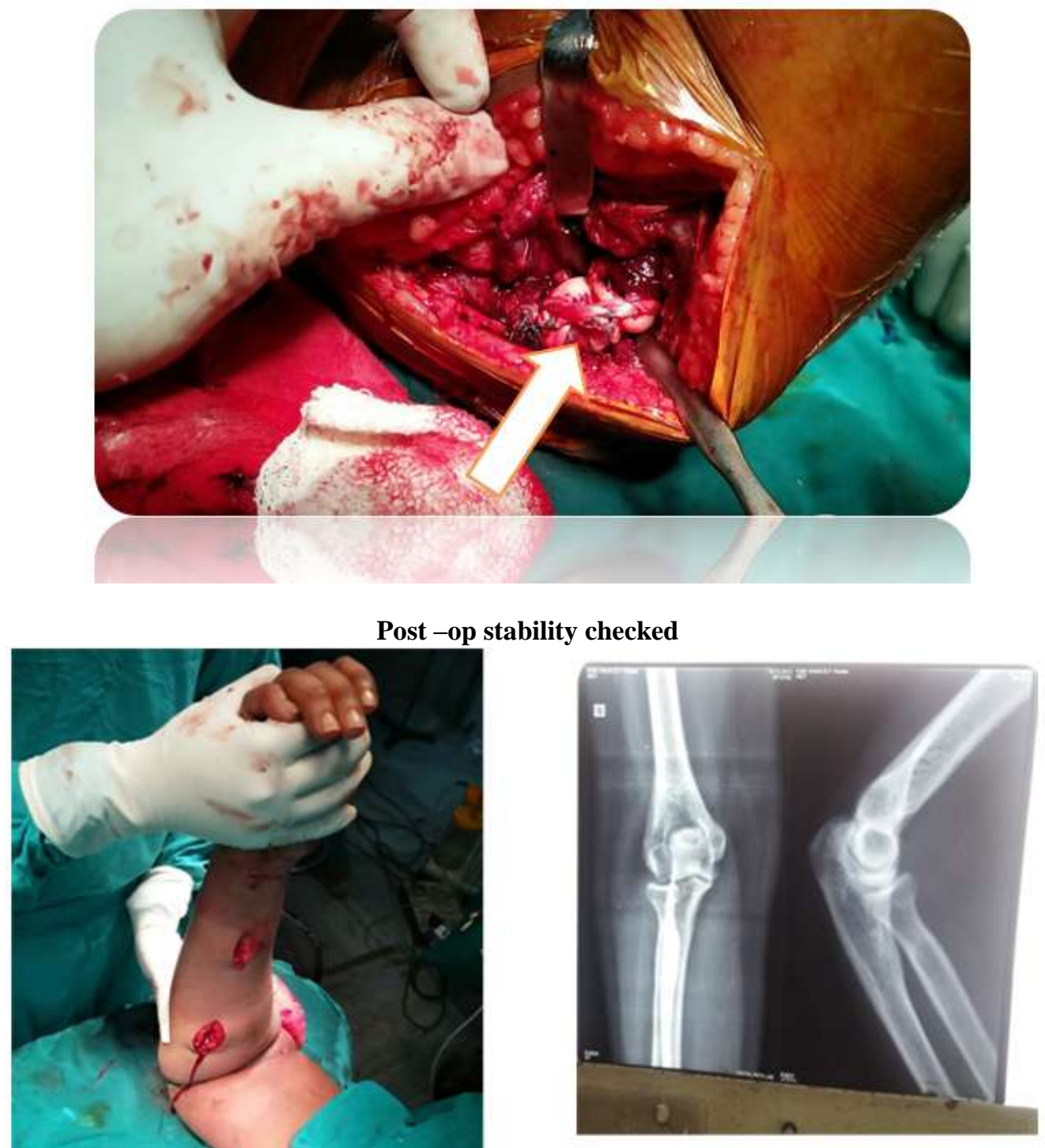


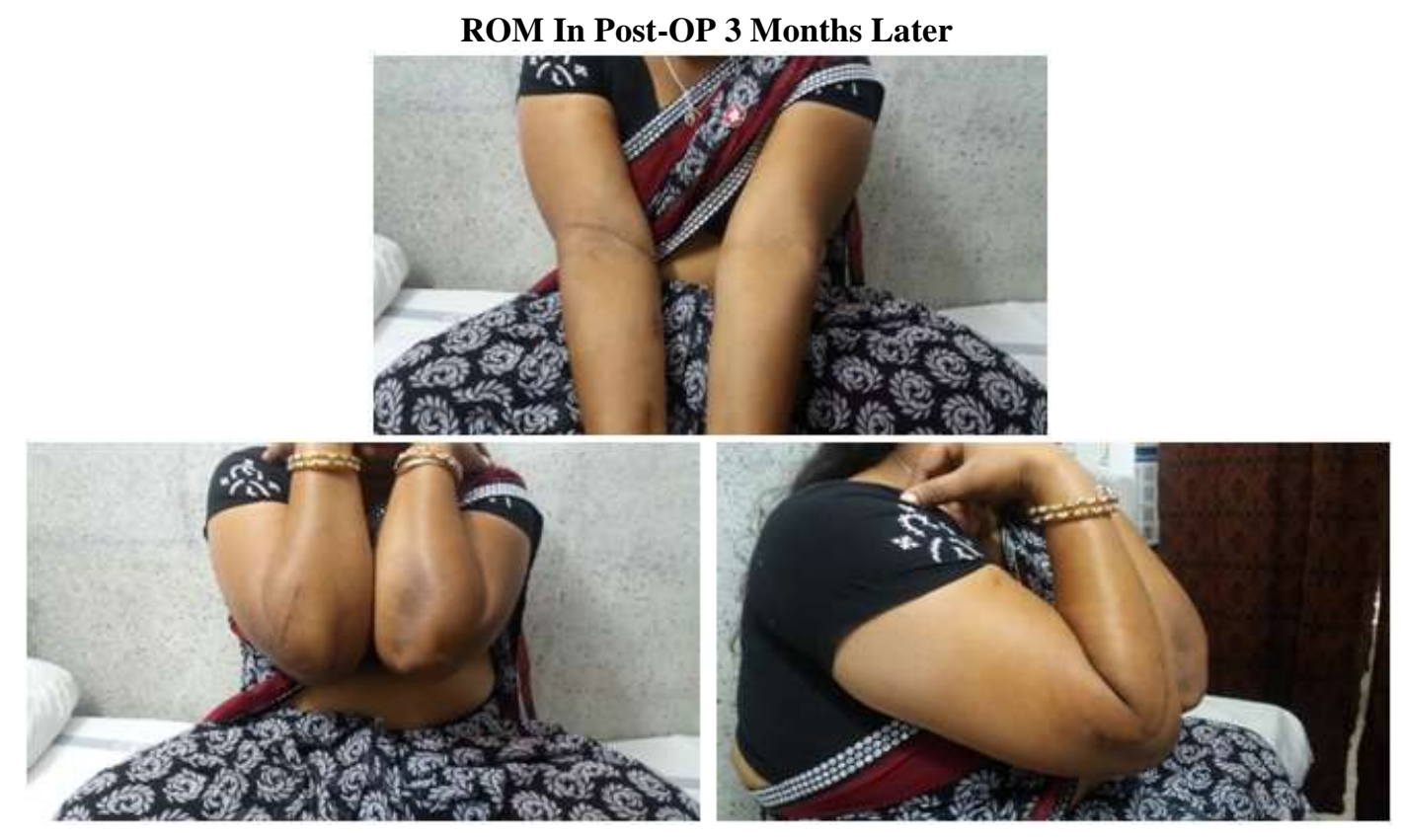

\section{Conclusion}

- As cases are reported to mostly young groups- High index suspicion, early diagnosis and treatment suggestive of good result and can prevent morbidity

\section{References}

[1]. Andrew JR, Timmerman LA. Outcome of elbow surgeries in professional baseball players. AM J sports 1995 JulAug 23(4): 407-13.

[2]. Paletta GA Jr. Wright GW. The modified docking procedure for elbow ulnar collateral ligament reconstruction. AM $J$ Sports med.2006 oct 34(10); 1594-8

[3]. king GJ, Dunning CE, Patterson SD, Jhonson JA. Single strand reconstruction of lateral collateral ligament restores varus and posterolateral stability of elbow. J Shoulder Elbow Surgery 2002 Jan-Feb 11(1); 60-4

[4]. Frasher JS, Pichora JE, Ferriera LM, Jhonson JA. Lateral collateral ligament repair rstores the varus stability of elbow. An invitro bio-mechanical study. J Orthop Trauma 2008 Oct 22(9):615-23

[5]. Lee M. Reichel, Graham S. Milam, MD Sean E. Sitton, MD Thomas L.Mehlhoff Elbow lateral colleteral ligament injury. Journal of American Society for Surgery of Hand January 2013 Volume 38, Issue 1, 184-201. 